\section{E-074 PREDICTORS OF HEMORRHAGIC CONVERSION FOLLOWING MECHANICAL THROMBECTOMY FOR ACUTE ISCHEMIC STROKE (AIS)}

A Boyke* , K Javed, J Dardick, I Naidu, J Ryvlin, D Kadaba, N Haranhalli. Montefiore Medical Center - Albert Einstein College of Medicine, Bronx, NY

\subsection{6/neurintsurg-2021-SNIS. 169}

Introduction Endovascular therapy is known to achieve a high rate or recanalization in patients with acute ischemic stroke (AIS) and is currently a standard part of emergent care. Hemorrhagic Conversion (HC) is a severe complication that may occur following ischemic stroke in patients who undergo a thrombectomy procedure. There is scarce data on the risk factors related to hemorrhagic conversion in patients who undergo mechanical thrombectomy. In our study, we sought to gain information on clinical characteristics and predictors associated with $\mathrm{HC}$ in patients treated with an endovascular intervention following AIS.

Methods We conducted a retrospective analysis of a population of adult patients treated with endovascular therapy for AIS at a primary stroke center in the Bronx, NY between January 2016 and February 2020. Bivariate and logistic regression analyses were performed to determine the predictors and impact on clinical outcome for patients who experienced Hemorrhagic Conversion, and for those who experienced symptomatic Hemorrhagic Conversion.

Abstract E-074 Table 1 Multiple logistic regression analysis model for all types of hemorrhagic conversion (10 predictor variables)

\begin{tabular}{lll}
\hline Independent Predictor & Adjusted OR (95\% Cl) & P value \\
\hline Age & $0.990(0.971-1.010)$ & 0.330 \\
Gender (male) & $1.621(0.865-3.038)$ & 0.132 \\
Prior Stroke & $3.230(1.532-6.811)$ & 0.002 \\
Hypothermia (Decreasing Temp ${ }^{\circ}$ F) & $1.503(1.084-2.083)$ & 0.015 \\
ASPECTS (<6) & $3.311(1.014-10.86)$ & 0.047 \\
TICI (2B-3) & $4.372(1.387-13.78)$ & 0.012 \\
IVtPA & $0.506(0.262-0.975)$ & 0.042 \\
Anesthesia (general) & $2.027(0.901-4.562)$ & 0.088 \\
Number of Passes (1) & $0.951(0.503-1.796)$ & 0.877 \\
Procedure Length (mins) & $1.002(0.994-1.006)$ & 0.967 \\
\hline
\end{tabular}

Abstract E-074 Table 2 Multiple logistic regression analysis model for symptomatic hemorrhagic conversion (10 predictor variables)

\begin{tabular}{lll}
\hline Independent Predictor & Adjusted OR (95\% Cl) & P Value \\
\hline Age & $.975(.931-1.02)$ & 0.29 \\
Gender (male) & $.852(.193-3.77)$ & 0.833 \\
Prior Stroke & $1.49(.353-6.29)$ & 0.587 \\
Hypothermia (Decreasing Temp ${ }^{\circ}$ F) & $.787(.391-1.59)$ & 0.503 \\
Aspects (<6) & $2.19(.272-17.62)$ & 0.461 \\
TICl (2B-3) & $.138(.028-.673)$ & 0.014 \\
IV tPA & $2.37(.583-9.62)$ & 0.228 \\
Anesthesia (general) & $1.95(.435-8.78)$ & 0.382 \\
Number of Passes (1) & $1.09(.257-4.61)$ & 0.908 \\
Procedure Length (mins) & $.999(.991-1.01)$ & 0.774 \\
\hline
\end{tabular}

Results A total of 325 patients who underwent endovascular therapy for AIS were identified. Of these, 74 (22.8\%) were observed to have hemorrhagic conversion, and $251(77.2 \%)$ had no hemorrhagic conversion. Of those diagnosed with hemorrhagic conversion, 25 (33.8\%) were found to be symptomatic. Bivariate analysis of presenting labs and vital signs showed no association with HC. In logistic regression analyses, a history of prior stroke (OR 3.23; 95\% CI 1.53-6.81), ASPECTS score <6 (OR 3.311; 95\% CI 1.01-10.86), and TICI 2B-3 (OR: 4.372; 95\% CI 1.39-13.78) were found to be independent predictors of hemorrhagic conversion. In the symptomatic hemorrhage group, a TICI (2B-3) recanalization (OR: $0.138 ; 95 \%$ CI .028-.673) was found to be the only independent predictor.

Conclusion History of prior stroke, radiographic evidence of infarct burden, and radiographic success of thrombectomy may be considered significant predictors for hemorrhagic conversion following AIS in patients who undergo mechanical thrombectomy.

Disclosures A. Boyke: None. K. Javed: None. J. Dardick: None. I. Naidu: None. J. Ryvlin: None. D. Kadaba: None. N. Haranhalli: None.

\section{A PROSPECTIVE, MULTI-CENTER, RANDOMIZED CONTROLLED PIVOTAL STUDY TO EVALUATE THE SAFETY AND EFFECTIVENESS OF TRUFILL ${ }^{\circledR}$ NBCA EMBOLIZATION OF THE MIDDLE MENINGEAL ARTERY FOR THE TREATMENT OF SUBDURAL HEMATOMA - THE MEMBRANE STUDY}

${ }^{1} \mathrm{C}$ Kellner*, ${ }^{2} \mathrm{~F}$ Al-Mufti, ${ }^{3} \mathrm{R}$ Gupta, ${ }^{4} \mathrm{~B}$ Jankowitz, ${ }^{5} \mathrm{R}$ Starke, ${ }^{6} \mathrm{~A}$ Rai. ${ }^{1}$ Neurosurgery, Icahn School of Medicine at Mount Sinai, New York, NY; ${ }^{2}$ Neurosurgery, Westchester Medical College at New York Medical, New York, NY; ${ }^{3}$ Neurology, Wellstar, Marietta, GA; ${ }^{4}$ Neurosurgery, Cooperhealth, Camden, Nj; ${ }^{5}$ Neurosurgery, University of Miami, Miami, FL; ${ }^{6}$ Neuroradiology, West Virginia University, Morgantown, WV

\subsection{6/neurintsurg-2021-SNIS.170}

Introduction Chronic subdural hematoma (cSDH) is one of the most common neurosurgical diseases with an incidence of 1-5.3 cases per 100,000 that increases significantly with age. When symptomatic, these lesions are often treated by surgery, an approach which does not directly address the underlying pathophysiology of chronic inflammation and neovascularization of the dura and has a high incidence of recurrence. Middle meningeal artery (MMA) embolization has emerged as a potential minimally invasive endovascular treatment option that may substitute for surgery or serve as an adjunct. TRUFILL n-BCA is potentially well suited as a liquid embolic in this procedure given that it is non-caustic, rapidly deployed, and able to be injected in a highly targeted fashion. Here we present the protocol for a randomized clinical trial to evaluate the safety and effectiveness of n-BCA embolization of the MMA for the treatment of cSDH.

Methods This investigational trial is a prospective, multi-center, open-label, randomized controlled study in which up to 376 subjects will be randomized to receive standard of care (SOC) alone or SOC and TRUFILL n-BCA MMA embolization for the treatment of cSDH. The study is designed to evaluate the effectiveness and safety of MMA in two cohorts - a surgical cohort and a non-surgical cohort. 